\title{
EXTRACTION AND IDENTIFICATION OF FORMULATIONS OF ANABOLIC ANDROGENIC STEROIDS: A FORENSIC EDUCATIONAL APPROACH
}

Lucas M. Berneira ${ }^{a}$, Marina Ritter ${ }^{a}$, Caroline C. da Silva ${ }^{a}$, Tais Polettia, Luan F. Passos ${ }^{a}$, Bruno N. da Rosa ${ }^{\text {a }}$, Marco A. Z. dos Santos ${ }^{\mathrm{a}}$ and Claudio M. P. de Pereira*,a,

${ }^{a}$ Centro de Ciências Químicas, Farmacêuticas e de Alimentos, Universidade Federal de Pelotas, 96900-010 Pelotas - RS, Brasil

Recebido em 24/03/2020; aceito em 22/06/2020; publicado na web em 07/08/2020

\begin{abstract}
The use of anabolic androgenic steroids is largely restricted worldwide since they are associated with innumerous side effects and health damage. However, apprehensions of formulations of anabolic agents have been extensively increasing throughout the world, making their analysis a common task in the forensic field. In this sense, a set of three experiments was developed and applied to undergraduate students of Forensic Chemistry dealing with visual inspection of apprehended formulations of anabolic androgenic steroids as well as chemical identification by Infrared Spectroscopy and Gas Chromatography-Mass Spectrometry. Moreover, the classroom evaluated the extraction of steroidal active ingredients by ultrasonic and microwave-assisted extraction. The experimental procedures allied with the theory behind these topics provided an important background to the students on distinct extraction methods and analytical techniques used in the analysis of anabolic agents. Finally, the classroom was led to critically evaluate and interpret the results as well as to question the adequacy of experimental methods used in forensic analysis to stimulate their critical thinking.
\end{abstract}

Keywords: chemical education; instrumentation; extraction procedures; forensic chemistry; anabolic androgenic steroids.

\section{INTRODUCTION}

Anabolic androgenic steroids (AASs) comprise a class of derivatives of testosterone that are known for their illicit use among athletes and the population in general in order to obtain a higher sportive performance and muscle mass. The abusive use of these substances is associated with several health hazards that include physical disorders, coronary damage, liver failure as well as neuropsychiatric problems. ${ }^{1,2}$ In this sense, formulations containing AASs are controlled or prohibited worldwide in order to regulate their distribution and consumption. ${ }^{3}$

Despite the measures to control the consumption of anabolic agents, there has been a considerable increase in apprehensions of formulations of AASs, which are mainly supplied by illicit manufacturers. Underground suppliers are not regulated by health agencies so their products are susceptible to chemical or biological contaminations, adulterations and falsifications, making the formulations significantly more dangerous to the health of the user. ${ }^{4}$ In Brazil, for instance, apprehension of AASs increased approximately $160 \%$ from 2009 to $2011 .^{3}$ Other researches have highlighted that counterfeit products account for about a third of the seized materials. ${ }^{5,6}$

Once apprehended, drug formulations have to be analyzed for their components to be detected by forensic professionals who can provide analytical evidences that are used in legal processes. ${ }^{7}$ In this sense, it is essential for undergraduate students to be introduced to common procedures and techniques used in the analysis of drugs given that these evaluations are common for a forensic expert. ${ }^{8}$ Moreover, reaction mechanisms, equipment features and extraction methods used in the analysis can be taught, leading to the understanding that the steps in the procedure may influence the results used in legal processes. ${ }^{9}$

Generally, analysis of apprehended drug formulations initiate with visual inspection for the observation of possible signs of falsification regarding aspects of the label, recipient and the internal content. ${ }^{10,11}$ The identification of the components of the sample can be achieved by

*e-mail: lahbbioufpel@gmail.com
Infrared Spectroscopy and Gas Chromatography-Mass Spectrometry (GC-MS). ${ }^{12,13}$ For GC-MS analysis, an extraction procedure is required, which is generally performed using an ultrasonic bath (UAE), however other methods can be used such as liquid-liquid or microwave-assisted extraction (MAE). ${ }^{6,14}$ Recently, microwaves have been applied to analyze samples since it is aligned with several principles of green chemistry. These procedures are usually employed in the analysis of suspected drug samples and can be easily performed in a classroom. ${ }^{15}$

As it can be seen, identification of samples is considerably important in the forensic field. Besides, the increasing number of apprehensions has been making these procedures a common task for forensic experts which must be performed with the appropriate methods in order to provide precise conclusions used in legal processes. ${ }^{3,16}$ Therefore, the aims of this work were to develop and evaluate an experimental procedure involving the analysis of two formulations of AASs employing visual inspection, Infrared Spectroscopy and GC-MS analysis using distinct forms of extraction (UAE and MAE) for an undergraduate class. Moreover, pedagogical goals were to teach several approaches to analyze formulations of AASs and the influence methods and techniques cause on the results of the analyses, stimulating the critical thinking of the students.

\section{EXPERIMENTAL SECTION}

\section{Reagents and materials}

For the experiments, HPLC-grade methanol (Sigma-Aldrich, Saint Louis, USA) was used. The Brazilian Federal Police kindly provided two formulations of AASs (Table 1) with nandrolone decanoate and testosterone propionate as the alleged active ingredients in the samples.

\section{Module I: Visual inspection}

Students were asked to evaluate the label and recipient containing the given formulations of AASs to analyze the presence, absence or 
Table 1. Information about the studied samples

\begin{tabular}{|c|c|c|c|c|}
\hline Structure & Supposed Active Ingredient & Exact mass $\left(\mathrm{g} \cdot \mathrm{mol}^{-1}\right)$ & Physical Aspect & Sample Name \\
\hline & Testosterone propionate & 344.24 & Dark yellow oil & Product \#1 \\
\hline & Nandrolone decanoate & 428.33 & Light yellow oil & Product \#2 \\
\hline
\end{tabular}

misspelled information such as active ingredient, trade name, name and address of the manufacturer as well as expiration and batch number (Figure 1). The recipient was also evaluated according to its suitability to contain the formulation.

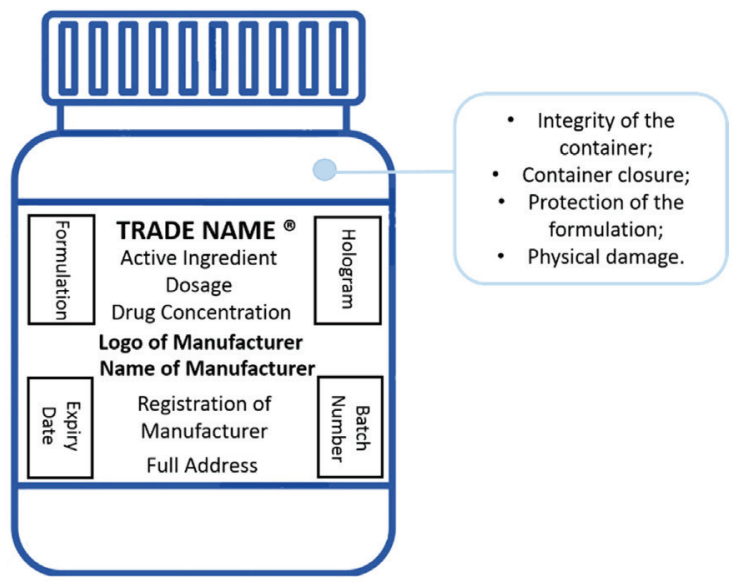

Figure 1. General aspects of visual inspection of questioned samples

\section{Module II: Infrared spectroscopy analysis}

One drop of each formulation was placed into the equipment and measurement was conducted in scan mode from $4000 \mathrm{~cm}^{-1}$ to $600 \mathrm{~cm}^{-1}$ with a resolution of $4 \mathrm{~cm}^{-1}$. After each analysis, the equipment was cleaned with acetone and a blank measurement was made.

\section{Module III: Extraction and GC-MS analysis}

Extraction of AASs was performed using UAE and MAE. For UAE, $25 \mu \mathrm{L}$ of the formulation and $5 \mathrm{~mL}$ of methanol were placed in conical tubes and vortexed for $10 \mathrm{~s}$. Subsequently, samples were immerged in an ultrasonic bath at room temperature for $10 \mathrm{~min}$ and centrifuged at $3000 \mathrm{rpm}$ for $5 \mathrm{~min}$. Finally, the methanolic fraction was transferred to vials and injected in the GC-MS. ${ }^{5}$

For MAE, $25 \mu \mathrm{L}$ of the formulation and $5 \mathrm{~mL}$ of methanol were placed in a round-bottom flask and mixed for $10 \mathrm{~s}$. The sample was introduced into the microwave equipment and subjected to $150 \mathrm{~W}$ of power for $10 \mathrm{~min}$. Afterwards, the methanolic portion was transferred to vials and analyzed by GC-MS.

\section{Instrumentation}

Spectroscopic analysis was performed on a spectrometer with Attenuated Total Reflection and Fourier Transform model Shimadzu
Prestige 21 (Shimadzu, Kyoto, Japan). For chromatographic analysis, one $\mu \mathrm{L}$ of the extracted material was injected into the equipment in split mode (1:25) into the GC-MS model QP2010SE (Shimadzu, Kyoto, Japan). The capillary column was Rtx-5MS (Restek, Bellefonte, USA) and the carrier gas was helium. The injection port and the transfer line both operated at $280{ }^{\circ} \mathrm{C}$ while the programed oven temperature was $200{ }^{\circ} \mathrm{C}$, increasing $30{ }^{\circ} \mathrm{C} \cdot \mathrm{min}^{-1}$ to $250{ }^{\circ} \mathrm{C}$ holding for $16 \mathrm{~min}$. Then, temperature was raised from $30^{\circ} \mathrm{C} \cdot \mathrm{min}^{-1}$ to $300{ }^{\circ} \mathrm{C}$ maintaining for $14.5 \mathrm{~min}$. MS had an electron ionization of $70 \mathrm{eV}$ and scanned from 30 to $550 \mathrm{~m} / \mathrm{z}$. The identification of the components of the samples was assessed by comparing the samples with the NIST-08 library included on the equipment software.

\section{Hazards and disposal}

Methanol is volatile and inhalation or contact can generate health damages, so it is recommended that students wear gloves and handle the solvent inside a fume hood. Throughout the extraction procedures, methanol can be volatilized and, thus, extraction equipment should be also placed inside a fume hood. Disposal of the methanolic solutions generated in the extraction procedures should be placed on the organic waste.

\section{RESULTS AND DISCUSSION}

The class had about $4 \mathrm{~h}$ of laboratory periods, which were divided into experimental modules and lectures about the techniques of identification and methods of extraction of formulations of AASs. Initially, one set of 10 undergraduate students were familiarized with the topics of the experiment by means of a $0.5 \mathrm{~h}$ expository lecture about steroids, mechanism of action and toxicology of anabolic agents, legislation of controlled medicines and analytical techniques for the analysis of formulations of AASs. Then, students performed Modules I and II in groups of three each, which took approximately $1.5 \mathrm{~h}$.

For Module III, students were divided in two groups that would perform UAE or MAE. It should be noted that, although not experimentally performing all the extraction procedures, the groups were asked to accompany both methods while they were made. Both extractions and injection in the GC-MS were executed in parallel lasting about $1 \mathrm{~h}$. As the samples were running in the equipment, there was another short lecture of $0.5 \mathrm{~h}$ about extraction methodologies for the analysis of AASs in order to expand the background of the students on the topic.

The combination of experimental and theoretical modules provided innumerous abilities to the students. The requirements for students in these experiments were to evaluate the veracity of 
a questioned formulation, detect the active ingredient and its other components and to compare the distinct extraction procedures in relation to the quantity of extracted analytes. The class was evaluated by means of student reports on the experiments and filling of a visual inspection checklist.

\section{Module I: Visual inspection}

Visualization of the elements in the label and the recipient were feasible tools in the screening of possible falsified products. For the analyzed materials, the alleged manufacturer did not have a health license to operate in Brazil, which means they were illegal medicines. ${ }^{6}$ Students observed that both samples displayed the trade name, active ingredient, logotype of manufacturer and expiry date. However, Product \#2 had other information in the label common to genuine medicines such as batch number, hologram, registration number and address of the manufacturer, which was not noted in Product \#1. Thus, visual inspection was able to initially provide signs of falsification in Product \#1 while Product \#2 was preliminarily designated as a genuine material.

In order to perform this exercise in the classroom, a checklist organized by the World Health Professions Alliance was given to the groups of students so that they could evaluate the label and packaging of the samples and observe possible signs of falsification or adulteration in the product. The products used in the analysis were real samples apprehended by the Brazilian Federal Police being genuine or falsified formulations. For practical purposes, the label could be developed by the teacher and inserted on a recipient to simulate apprehended samples. It would be interesting to pursue, if not existing, a partnership with local law-enforcement agencies, as they could play an important role in this type of experiment and provide further interaction of students with real case scenarios. The evaluation stimulated the students during the practical procedure since the proximity with reality made the experiment more interesting and easier to understand and correlate. Besides, it showed the importance of detailed observation and the professional responsibility that is associated with the analysis. It has been demonstrated that connection of theoretical classes with real-life situations is an important motivational method.

\section{Module II: Infrared spectroscopy analysis}

Infrared Spectroscopy analysis of the oily formulations (Figures 2 and $1 \mathrm{~S}$ ) revealed the presence of characteristic bands such as $=\mathrm{C}-\mathrm{H}$ $\left(3008 \mathrm{~cm}^{-1}\right),-\mathrm{C}-\mathrm{H}\left(2918 \mathrm{~cm}^{-1}\right), \mathrm{C}=\mathrm{O}\left(1743 \mathrm{~cm}^{-1}\right)$ and $\mathrm{C}-\mathrm{O}$ (1159 $\left.\mathrm{cm}^{-1}\right)$ in both samples. There were some differences on the spectra such as the presence of $\mathrm{CH}_{2}$ vibrations $\left(721 \mathrm{~cm}^{-1}\right)$ in Product \#1 and the presence of aromatic $\mathrm{C}=\mathrm{C}\left(1673 \mathrm{~cm}^{-1}\right)$ and $\mathrm{Ar}-\mathrm{H}\left(709 \mathrm{~cm}^{-1}\right)$ in Product \#2. Most of the vibrations could be associated to the alleged active ingredients in both samples, although the bands could be associated with excipients and other adulterants as well, leading to rather inconclusive results. In this sense, the use of Infrared Spectroscopy alone did not allow confirmation nor differentiation of samples, so that confirmatory analytical tools including chromatographic approaches were required in order to analyze the formulations.

The use of Infrared Spectroscopy was a feasible tool in order to introduce the student on the analytical techniques used in the detection of AAS. The spectroscopic analysis was a demonstration to the classroom that the determination of the active ingredient in a formulation can be a difficult task due to inferences of the matrix. ${ }^{17,18}$ In this sense, the use of a sequence of analytical techniques is favored in order to achieve precise conclusions about the nature of the sample. ${ }^{19}$ Moreover, the student could be familiarized with the instrumentation and manipulation of the equipment and interpretation of bands in the infrared spectra, which are important skills to their future careers.

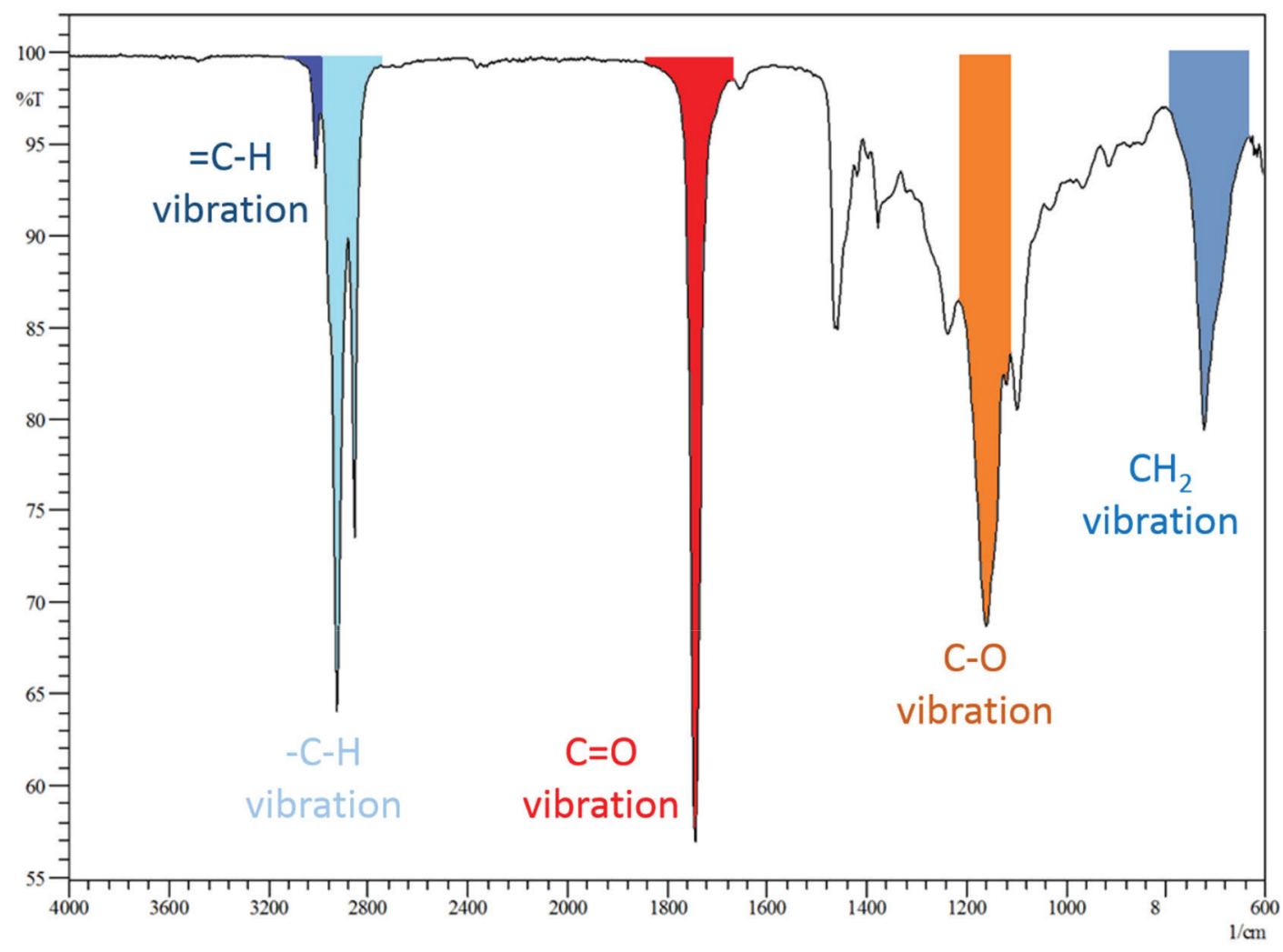

Figure 2. Infrared spectrum of the formulation of Product \#1 


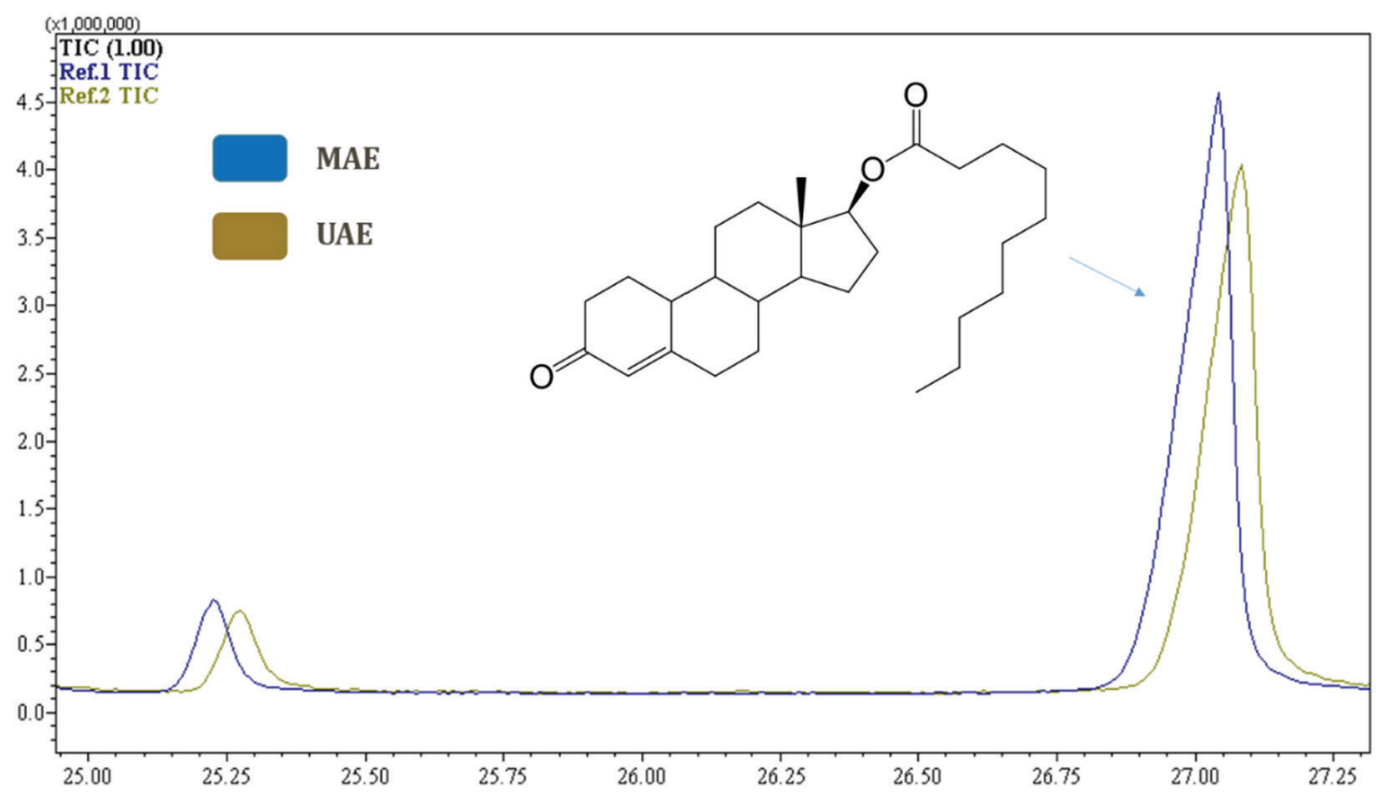

Figure 3. GC-MS chromatogram of MAE and UAE of methanolic extracts of the formulation of Product \#2

\section{Extraction and GC-MS analysis}

GC-MS analysis allowed the student to conclude that Product \#1 did not have its alleged active ingredient and was only composed of oily components. This conclusion was based on the lack of any peak in the chromatogram related to testosterone propionate, which placed the sample as a falsified material. This information was previously known and, therefore, Product \#1 was not used in the comparison of extraction procedures.

On the other hand, Product \#2 had its active ingredient detected (Figure 2S) and several excipients in the form of ethyl esters of fatty acids and benzyl benzoate (Figure 3S). Mass spectra fragmentation showed a molecular ion of $\mathrm{m} / \mathrm{z} 428$ and a base peak of $\mathrm{m} / \mathrm{z} 274$ which corresponds to the cleavage of the ester moiety bonded to the $17 \beta$ carbon. These fragments indicated the presence of nandrolone decanoate. Given that Product \#2 had the steroidal active ingredient it was used for the comparison of extraction procedures.

Comparison between the UAE and MAE (Figure 3) showed that the methods yield distinct extraction results of the sample constituents. Since analytical standards were not used in the procedure, the area of the peaks was used as an indicative of the concentration. Nandrolone decanoate had an area of 27,457.444 in $\mathrm{UAE}$ and 31,871.523 in MAE, indicating that microwave irradiation was more effective and that the type of extraction considerably influences on the overall results.

Regarding the proposed experiments, analysis of forensic samples by analytical techniques proved to be an important tool to enhance the learning of chemistry. On the other hand, results showed that several variables could affect the extraction process leading to the conclusion that the results should be carefully interpreted. Differences among the results can be related to the distinct mechanisms of extraction of UAE and MAE. ${ }^{20,21}$ Finally, the students could observe the importance of conducting an adequate sample preparation and that the development and evaluation of novel procedures of extraction are important in order to obtain better results.

\section{CONCLUSIONS}

Analysis of AASs formulations was successfully used to immerge undergraduate students in the routine of a forensic chemist, showing the importance regarding the choice of methodology, data analysis and critical thinking. Students were able to be related to subjects including AASs, extraction and analytical techniques used in the analysis. Moreover, students observed that the analysis required several analytical tools in order to obtain adequate results. In conclusion, comparison between extraction procedures highlighted that the results should be carefully interpreted since in real cases the conclusions generated by the forensic expert about an unknown sample affect legal processes.

\section{SUPLEMENTARY MATERIAL}

The Supplementary Material section shows analytical data of the analyzed formulations and is freely available at http://quimicanova. sbq.org.br in pdf format.

\section{ACKNOWLEDGMENTS}

The authors are thankful to the Brazilian Federal Police, INCT (Grant number 465450/2014-8), CNPq and FAPERGS.

\section{REFERENCES}

1. Kicman, A. T.; Br. J. Pharmacol. 2008, 154, 502.

2. Solimini, R.; Rotolo, M. C.; Mastrobattista, L.; Mortali, C.; Minutillo, A.; Pichini, S.; Pacifici, R.; Palmi, I.; Eur. Rev. Med. Pharmacol. Sci. 2017, 21,7 .

3. Neves, D. B.J.; Marcheti, R. G. A.; Caldas, E. D.; Forensic Sci. Int. 2013, 228, e81

4. Thevis, M.; Schrader, Y.; Thomas, A.; Sigmund, G.; Geyer, H.; Schänzer, W.; J. Anal. Toxicol. 2008, 32, 232.

5. Neves, D. B. J.; Caldas, E. D.; Forensic Sci. Int. 2017, 275, 272.

6. Berneira, L. M.; de Freitas, S. C.; da Silva, C. C.; Machado, A. de M.; de Pereira, C. M. P.; dos Santos, M. A. Z.; Forensic Sci. Int. 2019, 296, 15.

7. Schurter, E. J.; Zook-Gerdau, L. A.; Szalay, P.; J. Chem. Educ. 2011, 88, 1416.

8. Parker, P. D.; Beers, B.; Vergne, M. J.; J. Chem. Educ. 2017, 94, 1522.

9. Garcia Sega, E.; Clarke, J.; J. Chem. Educ. 2013, 90, 1658.

10. Kozlik, P.; Tircova, B.; Steroids 2016, 115, 34. 
11. Pereira, T. M. C.; Júnior, J. A. Q.; Ortiz, R. S.; Rocha, W. F. C.; Endringer, D. C.; Filgueiras, P. R.; Poppi, R. J.; Romão, W.; Anal. Methods 2014, 6, 2722.

12. Anderson, C.; J. Chem. Educ. 2009, 82, 1809.

13. Werner, T. C.; J. Chem. Educ. 2012, 89, 624.

14. de Freitas, S. C.; dos Santos, M. A. Z.; Berneira, L. M.; Ortiz, R. S.; de Pereira, C. M. P.; Sci. Justice 2018, 59, 190.

15. Timmer, B. J. J.; Schaufelberger, F.; Hammarberg, D.; Franzén, J.; Ramström, O.; Dinér, P.; J. Chem. Educ. 2018, 95, 1301.

16. Berneira, L. M.; dos Santos, M. A. Z.; da Silva, C. C.; Passos, L. F.; Ortiz, R. S.; Machado, A. de M.; de Pereira, C. M. P.; Chem. Pap. 2020
17. Chiong, D. M.; Consuegra-Rodriguez, E.; Almirall, J. R.; J. Forensic Sci. 1992, 37, 13257.

18. Siddiqui, M. R.; Alothman, Z. A.; Rahman, N.; Arabian J. Chem. 2017, 10, 1409 .

19. Krug, O.; Thomas, A.; Walpurgis, K.; Piper, T.; Sigmund, G.; Schänzer, W.; Laussmann, T.; Thevis, M.; Eur. J. Clin. Pharmacol. 2014, 70, 1303.

20. Yang, Y.; Li, H.; Zhang, J.; Sun, N.; Food Anal. Methods 2014, 7, 798.

21. Galesio, M.; Mazzarino, M.; De La Torre, X.; Botrè, F.; Capelo, J. L.; Anal. Bioanal. Chem. 2011, 399, 861. 